\title{
marges Marges
}

revue d'art contemporain Revue d'art contemporain

$20 \mid 2015$

Dispositif(s) dans l'art contemporain

\section{Following Piece : l'a posteriori du dispositif indiciel}

Following Piece : a posteriori of an indexical "dispositif"

\section{Sophie Lapalu}

\section{OpenEdition}

Journals

Édition électronique

URL : http://journals.openedition.org/marges/987

DOI : $10.4000 /$ marges.987

ISSN : 2416-8742

\section{Éditeur}

Presses universitaires de Vincennes

\section{Édition imprimée}

Date de publication : 1 mars 2015

Pagination : 103-118

ISBN : 978-2-84292-424-9

ISSN : $1767-7114$

Référence électronique

Sophie Lapalu, «Following Piece : I'a posteriori du dispositif indiciel », Marges [En ligne], 20 | 2015, mis en ligne le 01 mars 2017, consulté le 01 mai 2019. URL : http://journals.openedition.org/marges/987 ; DOI : $10.4000 /$ marges. 987 



\section{Following Piece: l'a posteriori du dispositif indiciel}

En 1969, le jeune poète qu'était Vito Acconci décida non plus d'écrire un poème consacré à l'action de suivre, mais d'engager son corps quotidiennement, durant vingt-trois jours, dans une filature. Intitulée Following Piece, cette action est aujourd'hui considérée comme un tournant décisif dans la carrière de l'artiste, un véritable saut hors de la page et de la poésie au profit de l'usage du corps dans l'espace réel. Acconci ne se contenta pas cependant d'agir furtivement, sans être perçu et créa un ensemble d'éléments relativement hétérogènes lui permettant de rendre publiques ces actions nécessairement secrètes. Quels sont ces éléments? Quel ensemble constituent-ils, et comment fonctionnent-ils? Enfin, à qui s'adressent-ils? Dans un premier temps, nous replacerons Following Piece dans son contexte afin de mieux saisir ce qui incita l'artiste à agir ainsi. Nous étudierons par la suite les différents moyens mis en place par Acconci afin de faire connaître cette série de filatures auprès d'un public de l'art et tenterons d'en saisir le mode de fonctionnement, pour enfin proposer une définition de l'ensemble constitué.

Cette œuvre voit le jour à la fin des années 1960 à New York, dans un contexte artistique marqué par un désir profond de se désaliéner du pouvoir de l'institution, de la galerie ou de la presse spécialisée - tendant même à vouloir se débarrasser d'une "scène » de l'art au profit d'un contact direct avec le public, par exemple dans des espaces autogérés/1. L'ultime année de la décennie fut particulièrement riche en bouleversements et vit naître notamment The Art Workers'Coalition/2, mouvement engagé contre les pratiques muséales. Fondé notamment par le critique d'art John Perreault, ce dernier organisa la
/1 On pense par exemple au projet de George Maciunas de créer une coopérative Fluxus dans les anciens locaux industriels de Soho dès 1966, ou à la revue $o$ to 9 cofondée par Vito Acconci et Bernadette Mayer l'année suivante.

/ $\mathbf{2}$ Le mouvement est également destiné à défendre les droits des femmes, les droits civiques et le refus de la guerre du Vietnam. Pour plus d'informations sur le groupe, voir Julia Bryan-Wilson, Art Workers: Radical Practice in the Vietnam War Era, Berkeley, University of California Press, 2009.

\section{Ci-contre}

Affiche pour l'exposition et les événements de " Street Works IV », 1969. 
/3 John Perreault, « Taking to the Street ", Village Voice, 16 octobre 1969, p. 15 . Nous traduisons.

/4 «Street Works I »: 24 heures du 15 mars 1969, block dessiné par Madison, $5^{\mathrm{e}}$ et $6^{\mathrm{e}}$ avenues, $42^{\mathrm{e} j u s q u}$ 'à la $52^{\mathrm{e}}$ rues; "Street Works II » : entre 17 heures et 18 heures le 18 avril, block formé par les $5^{\mathrm{e}}$ et $6^{\mathrm{e}}$ avenues et les $13^{\mathrm{e}}$ et $14^{\mathrm{e}}$ rues. "Street Works III » : 25 mai, de 9 heures à minuit, de Prince street à Grand street, de Wooster street à Greene street (soit un périmètre bien plus large).

/5 John Perreault, cité par Anna Dezeuze, «In search of the Insignificant, Street Work, "Borderline, Art and Dematerialisation" ", dans lleana Parvu (sld), Objets en procès, Après la dématérialisation de l'art 1960-2010, Genève, MetisPresses, 2012, p. 35. Nous traduisons.

/6 Ce chiffre correspond en fait au nombre d'invitations envoyées par les organisateurs. Voir Lucy Lippard, Six Years: Dematerialization of the Art Object (1973), Berkeley, University of California Press, 1997, p. 90, 94, 102, 117.

/7 Organisation à but non lucratif.

/8 Cette institutionnalisation fut aussi provoquée par un incident raconté dans John Perreault, « Paravisual ", Village Voice, 5 juin 1969, p. 17. même année, avec Hanna Weiner et Marjorie Strider, les cinq occurrences d'un projet qui nous intéresse ici, sobrement intitulé "Street Works ». Série de manifestations new-yorkaises, Perreault les définit comme suit: « (1) Un Street Work a lieu dans la rue; (2) un Street Work perd son effectivité si transféré à l'intérieur d'une galerie ou d'un musée; (3) un Street Work est temporaire ; (4) un Street Work est prévu, identifié, ou créé par un artiste/3. ». Les artistes agirent donc à leur guise, dans le cadre d'une temporalité donnée et d'un espace désigné/4. La troisième manifestation fut d'ailleurs ouverte à tous les participants; impossible d'en connaître précisément le contenu. Perreault le reconnaît: "Il était difficile de dire quels objets et quelles activités étaient ou n'étaient pas des Street Works/5. ». Toute activité prenant place dans la rue était susceptible d'être un "Street Work»; les propositions furent si diffuses et imperceptibles que le chiffre sept cents fut avancé pour les dénombrer/6!

Malgré la défiance à l'égard des institutions, la quatrième manifestation fut sponsorisée par l'Architectural League of New York/7, les participants sélectionnés et un vernissage organisé le 2 octobre, au numéro $41 \mathrm{Est}$ de la $65^{\mathrm{e}}$ rue. Ce qui avait été initié de manière presque spontanée, ce qui était ouvert à tous les participants dans un désir de liberté, se trouva récupéré en moins d'une année par un organisme institutionnel/8. L'affiche de cet événement stipulait: "Travaux de chacun des onze artistes, suivis par des œuvres dans divers endroits jusqu'au 25 octobre/9. ». Nous ne savons pas si une exposition plus classique était également présentée, mais il est certain qu'une programmation d'événements marquant le commencement avait été organisée "durant toute la journée du 2 octobre », comme le laisse penser Alphabetical T-shirts de John Perreault - œuvre qui consista à inscrire une des lettres de l'alphabet sur vingt-six tee-shirts portés par autant de personnes lors du vernissage de "Street Works IV ». Vito Acconci, lui, se planta deux heures sur un trottoir pour regarder la circulation/10.

Les propositions qui coururent jusqu'à la fin du mois s'inscrivirent pour leur part dans la lignée des précédentes: il était toujours impossible, pour le passant lambda, de distinguer une proposition artistique d'une activité urbaine quelconque.

Vito Acconci fut sélectionné pour cette quatrième manifestation suite à sa participation aux trois premiers "Street Works ». Entraîné par une écriture qui déborde les limites du champ poétique, les actes se substituent aux mots: une fois par jour, durant vingt-trois jours, il choisit au hasard une personne dans la rue pour la suivre jusqu'à ce qu'elle entre dans un lieu privé. Ainsi, au lieu d'écrire un poème consacré à l'action de suivre, Acconci engage son corps dans la fila- 
ture: «Pour moi, Following Piece était une façon, une façon littérale, de quitter ma carrière de poète. Il le fallait. D'une certaine manière, la poésie est quelque chose que l'on fait chez soi. On s'assoit à sa table, on écrit dans son bureau. Pour ne pas rester dans cette pièce, j'avais la solution de sortir en ville. Pour avoir une raison de sortir en ville, je pouvais décider de me concentrer sur une personne dans la rue et laisser cette personne me guider dans la ville/11. ".

L'action pouvait durer quelques minutes ou plusieurs heures, selon les espaces utilisés par la personne suivie. Tant que cette dernière se rendait dans un magasin ou un cinéma, Acconci la suivait. Lorsqu'elle entrait dans un immeuble, alors l'activité prenait fin. Si le postulat des "Street Works » énonçait l'engagement dans les avenues newyorkaises lors d'un temps assigné, le contrat était parfaitement rempli: toutes les rues de la ville, toutes les secondes du mois étaient potentiellement allouées à l'expérience qu'il se proposait de vivre.

Mais quelle invitation fut faite aux spectateurs? Aucun d'entre eux n'était convoqué et il était impossible de différencier l'action artistique d'un déplacement quotidien. Following Piece, irréductible à l'appréhension traditionnelle d'une œuvre, ne fut pas perçue par un public lors de son accomplissement. Quelle stratégie Vito Acconci établit-il afin que son œuvre ne se dissolve pas dans son effectuation?

\section{Actions furtives}

Rien ne permettait de désigner le caractère artistique de Following Piece et pour cause : comment réussir une filature si ce n'est en passant inaperçu? L'adjectif furtif/12, qui caractérise l'aspect fugace et éphémère des objets auquel il est associé, qualifie bien cet acte. Il désigne en effet ce qui est secret, caché intentionnellement, soit, ici, la double nature de l'action: en tant que filature, elle nécessite d'être clandestine pour réussir; en tant que proposition artistique, elle serait invalidée par la convocation de spectateurs qui en empêcheraient l'accomplissement.

S'il est entendu que Following Piece prend place dans un cadre offert par Perreault, ce cadre est invisible. Au contraire du musée où, pour reprendre les mots de l'artiste, "tout ce qui était séparé devenait automatiquement objet d'attention/13 », le spectateur de "Street Works » ne pouvait que déambuler à son tour, flâner sans «focalisation préétablie », dans «le monde désordonné lui-même/14 ». En conséquence, nous pouvons affirmer que ses poursuites n'ont pas bénéficié de spectateur. Ce fut vraisemblablement une des raisons pour lesquelles Vito Acconci refusait de parler de performance, au profit du terme «activité/15 ». Il est en effet généralement considéré
/9 cf. affiche reproduite dans o to 9, op. cit., n.p.

/10 Voir Vito Acconci: Diary of a Body, 19691973, Charta, Milan, New York, 2006, p. 74. Nous traduisons.

/11 Vito Acconci, cité dans Mela Davila (sld), Vito Hannibal Acconci Studio, catalogue d'exposition (Nantes, Musée des Beaux Arts, Barcelone, MACBA, 2005), Barcelone, MACBA, 2004, p. 9.

/12 Nous reprenons la terminologie de Patrice Loubier. Patrice Loubier et Anne-Marie Ninacs (sld), Les commensaux, quand l'art se fait circonstances, SKOL, Centre des arts actuels, Montréal, 2001.

/13 Vito Acconci, " "Performance"... a posteriori », Documents sur l'art contemporain, mars $1992, n^{\circ}$ o, p. 44.

/14 ibid., p. 45.

/15 « L'art selon moi devrait être considéré comme une activité. Et plus précisément comme une activité égale à toutes les autres. » cité dans Mela Davila (sld), op. cit., p. 26. 
/16 RoseLee Goldberg l'affirme, dans son ouvrage dédié à la discipline : "La performance est une façon d'appeler directement le public, de heurter l'auditoire pour l'amener à réévaluer sa propre conception de l'art et de ses rapports avec la culture.». RoseLee Goldberg, La Performance du futurisme à nos jours (1978), trad. C.-M. Diebold et L. Echasseriaud, Paris, Thames \& Hudson, 2001, p. 8.

/17 Vito Acconci, " "Performance"... », op. cit., p. 46.

/18 cf. Vito Acconci et Bernadette Mayer (sld), op. cit.

/19 Vito Acconci: Diary of a Body, 1969-1973, Milan, Charta, 2006, p. 79.

/20 « Mais l'action n'a pas été faite comme une activité privée (il n'y avait pas de transcription de mes interprétations, mes sentiments, mon expérience subjective) mais comme un exemple, un modèle; il y avait la liste des faits. L'action a été faite, depuis le début, de façon à ce qu'elle puisse être transformée en reportage, en rumeur: l'action, qui a commencé à partir d'un mot (schéma, phrase), a été faite seulement pour revenir aux mots. » Vito Acconci, Vito Acconci, op. cit., p. 10. que la performance limite la distance entre le performeur et le public, artiste et spectateur vivant l'œuvre simultanément/16. Acconci n'élimine pas tant la distance que le spectateur lui-même: impossible de parler d'auditoire puisqu'il s'ignore en tant que tel.

Following Piece présente toutefois une contradiction: si une filature réussie se doit d'être furtive intrinsèquement, en tant qu'activité artistique, peut-elle rester cachée? Ce paradoxe interpelle bien évidemment l'artiste qui, rétrospectivement, analyse ainsi la situation: "Soit la performance se mélangeait au décor et elle était alors à peine remarquée; soit elle était tellement extraite de son environnement que l'acteur pouvait faire figure de délinquant ou de cinglé. Dans l'un et l'autre cas, la performance recherchait la lumière/17. ".

L'artiste a nécessairement besoin de la reconnaissance d'un public afin de faire advenir son activité comme proposition symbolique. Pour ne pas rester le fou anonyme qui suit les New-yorkais dans la rue, son activité secrète doit fatalement être révélée.

\section{Une suite à la poursuite}

Paru en juillet 1969, le numéro 6 de la revue $o$ to 9/18 - fondée par Vito Acconci et Bernadette Mayer - est augmenté d'un supplément qui présente la documentation fournie par les artistes des trois premières manifestations de "Street Works ». Mais la revue s'arrêta à ce numéro et la nécessité de trouver un autre moyen de publicisation apparut. II n'est pas surprenant ainsi d'annoncer qu'il y eut une suite à la filature (a follow up to following piece): chaque jour, durant le mois qui suivit sa participation à "Street Works IV », Acconci envoya une lettre à une personne quelque part à New York ("City» Series). Ces missives décrivent les détails particuliers de l'épisode qui s'était produit un mois auparavant. Par exemple:

" 11 octobre

$15 \mathrm{~h} 44,8^{\mathrm{e}}$ rue et $6^{\mathrm{e}}$ Avenue, à l'angle nord-est

Femme en manteau orange: elle marchait vers l'est sur la $8^{\mathrm{e}}$ rue, côté nord de la rue

À 15 h 57, elle entre dans le magasin de cuir Fred Braun.

À $16 \mathrm{~h} 18$, elle quitte Fred:? Braun et continue à l'est.

À $16 \mathrm{~h} 29$, elle entre dans Experiment one, un magasin de vêtements.

À $16 \mathrm{~h} 52$, elle quitte Experiment one et marche à l'ouest [...]/19 ".

L'ensemble forme un catalogue des activités motrices d'Acconci et décrit factuellement le sujet humain dans son environnement (l'heure et les lieux sont donnés), telle une tentative objective de circonscrire Following Piece. Partant d'un mot ("suivre »), l'action retourne ainsi aux mots/20. 
Un exemplaire envoyé à Seth Siegelaub le 22 novembre 1969 est conservé aujourd'hui par le MoMa (Private Piece for Seth Siegelaub, novembre 1969) et un entretien filmé/21 réalisé en 1973 par le fondateur de la revue Avalanche, Willoughby Sharp, constituent de rares documents attestant de ces envois. Dans l'entretien, au travers des nuages de fumée de cigarette, Vito Acconci explique au critique d'art pourquoi il lui a adressé une de ces lettres. En sélectionnant ses destinataires selon des critères élitistes d'appartenance au monde de l'art (il cite en exemple Robert Morris), il espère que sa filature sera reconnue artistiquement. Ce monde de l'art serait le seul contexte où, d'après lui, ce type de travail peut être révélé et avoir une place. Ainsi semble résolue la question de l'accès public à une pièce a priori privée. Il y eut toutefois une « suite à la suite »: en décembre 1969, le même procédé d'envoi s'appliqua, en étant étendu au pays entier (avec les «Nation » Series). La lettre envoyée en novembre, ainsi que la description de l'épisode accompli en octobre, y étaient spécifiées. Plus tard, Acconci étendit sa méthode au monde entier : une feuille dactylographiée, conservée à la Bibliothèque du Musée des Beaux-Arts du Canada, présente un système plus complexe encore. En guise d'entête, figure le titre Private Piece for David Askevold et l'expression " "World" Serie ", puis la formule:

"Suivi d'une réactivation d'une réactivation d'une situation d'activité utilisant la rue, les voyages, la filature, le changement de position, "le fait d'aller partout où vous allez"/22. ".

Le protocole général de Following Piece est énoncé, puis l'activité du 4 octobre 1969 suivie de ses trois réactivations à un mois d'intervalle chacune, toutes dédiées à une personnalité différente.

« 4 novembre: L'activité particulière est réactivée pour Robert Barry ("City" Serie).

4 décembre : L'activité particulière et sa réactivation précédente sont réalisées pour Robert Ashley (" “Nation” Serie »).

4 janvier 1970: L'activité particulière et ses deux réactivations précédentes sont refaites pour David Askevold./23 ".

Pourquoi ces trois hommes? Probablement une fois encore pour leur rôle au sein du monde de l'art nord-américain. L'artiste David Askevold était professeur depuis 1968 au Nova Scotia College of Art and Design d'Halifax (Nouvelle Écosse, Canada)/24, Robert Ashley avait fondé le festival annuel ONCE dédié aux performing arts à Ann Arbor (1961-1969), avant de diriger le Center for Contemporary Music au Mills College (Oakland), tandis que Robert Barry était un des représentants majeurs de l'art conceptuel new-yorkais.

Ce document nous révèle le caractère systématique et organisé d'Acconci, qui semble catégoriser ses envois : titre, date, description
/21 Willoughby Sharp, «Willoughby Sharp Videoviews Vito Acconci », 1973 (http://www.ubu. com/film/acconci_sharp. html), consulté le 14 octobre 2013.

/ 22 Vito Acconci, Private Piece for David Askevold ("World » Serie), Ottawa, Bibliothèque du Musée National des Beaux Arts du Canada, 1969. Nous traduisons.

/23 ibid.

/24 Il avait fondé la Project Class, où des artistes, souvent issus de la sphère conceptuelle, étaient invités à proposer une œuvre à réaliser, compléter ou produire avec les étudiants. 
/ 25 Le 5 novembre 1969, On Kawara était d'ailleurs à New York et envoya sa carte postale à Lucy Lippard, qui vivait alors à SoHo. Lucy Lippard, op. cit., p. 125.

/ 26 Dont la figure tutélaire, Ray Johnson, vécut à New-York jusqu'en 1968.
/ 27 Voir l'exposition imaginée par Mel Bochner: «Working Drawings And Other Visible Things On Paper Not Necessarily Meant To Be Viewed As Art ", qui eut lieu en 1966 à la School of Visual Arts de New York.

y sont minutieusement inscrits, ainsi que leur classification ("City» Serie, etc.). Les envois répondent toujours d'une logique de déploiement (de la ville, au pays, à l'international) et forment une série qui conjugue distance temporelle (la date d'envoi est de plus en plus éloignée de l'activité « originelle» du mois d'octobre 1969) et distance spatiale (entre New York et le lieu de réception de la lettre).

Très factuelles, il n'est jamais question de ressenti, d'interprétation ou d'expérience intime. Ces lettres, qui présentent plutôt un paradigme de filature, tendent vers le général. Elles ne sont pas sans rappeler le caractère systématique et administratif des cartes postales envoyées quotidiennement par On Kawara, où l'heure à laquelle l'artiste s'est levé et l'adresse où il se trouve sont inscrites/25 et paraissent se situer ainsi au point de rencontre de différents courants new-yorkais de l'époque, comme le mail art/26 et l'art conceptuel/27.

Acconci étendit encore son champ d'action. Le Metropolitan Museum of Art de New York possède en effet une série de quatre photographies noir et blanc, représentant deux hommes marchant dans la rue, l'un suivant l'autre à une distance rapprochée. En raison de leur imagerie séquentielle, les travaux imitent à la fois l'apparence et la logique de cadres filmiques, la photographie du mouvement de Muybridge, mais surtout, ce qui parait bien logique dans ce cas, les photographies de filatures exécutées par la police ou les détectives privés. Disposées en quadrilatère, elles sont à lire dans les sens des aiguilles d'une montre et donnent une impression de mouvement continu (les deux images d'en haut semblent prises à quelques secondes d'intervalles, tandis que les hommes s'approchent de l'objectif). Les images du bas présentent les hommes de dos, filés par le photographe. Grâce à sa coiffure particulière, nous reconnaissons Vito Acconci: il accomplit une filature. Ces photographies s'ajoutent donc aux lettres comme procédé de publicisation. Étaient-elles destinées à être exposées dans un musée comme aujourd'hui, ou envoyées à une personne privée? Au dos d'un des clichés, sur le carton de montage, est inscrit: « PLEASE RETURN TO :// VITO ACCONCI // 39 PEARL STREET // BROOKLYN, NY $11201 \%$.

Ceci nous laisse supposer que ce tirage fut expédié. De plus, si la fiche technique du Met stipule que ces photographies datent de 1969, elle précise qu'elles sont signées et datées de 1970, ce qui soulève de nombreuses questions: quelle est la date de la prise de vue photographique? Quand a été réalisé le tirage?

Enfin, que considère conserver le Metropolitan Museum? L'action (datée de 1969) ou la photographie (de 1970 apparemment) ? Deux de ces images sont également reproduites au sein d'un panneau d'une taille de $76 \times 100,6 \mathrm{~cm}$ et divisé en 25 parties. Conservé au MoMA, il 
est constitué de 23 fiches de carton disposées bord à bord. Sur chacune d'elles est inscrit manuellement, à l'encre, le détail d'un jour de filature. Cet assemblage est complété par les deux photographies présentant les hommes de dos. Au fronton et au pied de l'ensemble est inscrit à la craie, « FOLLOW DIFFERENT PERSON EVERY DAY UNTIL PERSON ENTERS PRIVATE PLACE » [suivez une personne différente chaque jour jusqu'à ce que cette personne entre dans un espace privé]; puis dans l'angle inférieur droit, l'artiste a signé et inscrit le titre et le contexte de la filature. Ce panneau est donc parfaitement explicite: les fiches sont rangées par ordre chronologique, les filatures « illustrées» et les codes alloués à une œuvre d'art sont repris (titre, signature, date et même sponsor). Selon un même principe, Vito Acconci participa à l'élaboration de l'ouvrage Diary of a Body/28, paru en 2006: six pages sont consacrées à Following Piece. On y retrouve agencées les quatre photographies, les comptes rendus tapés à la machine (qui diffèrent légèrement de ceux écrits à la main), des notes sur ce travail, parfois largement postérieures et un schéma démontrant que l'artiste comme les passants suivis se dirigent vers le même but. La démonstration quant à la réalisation de Following Piece est complète.

Actions privées accomplies dans la sphère publique, les filatures s'arrêtent a priori au domaine privé de la personne suivie et à l'espace temporel de "Street Works IV ». Cependant, les comptes rendus, adressés de façon privée à des personnalités choisies pour leur image publique et les photographies et tableaux, destinés à être exposés, étendent l'espace et le temps de l'acte furtif. Ces artefacts, créés a posteriori, semblent ainsi former, avec l'action, un ensemble structurel, dont le déroulement du premier geste ne formerait pas le tout.

\section{Un dispositif indiciel}

Nous nommerons "dispositif » cet ensemble hétérogène de lettres, de photos, de panneaux qui permettent de rendre publique l'action privée et nous autorise à désigner ainsi les différentes publicités. Celles-ci s'inscrivent dans une double relation, à savoir avec ce qu'elles (re)présentent d'une part et avec leur récepteur d'autre part. Pour tenter de saisir le fonctionnement de ces relations, nous nous référons à la sémiotique de Charles Sanders Peirce, qui élabora une science des signes en fonction de leur activité de relation.

À la question "Qu'est ce qu'un signe? », Peirce répond: "Un signe ou representamen, est quelque chose qui tient lieu pour quelqu'un de quelque chose sous quelque rapport ou à quelque titre. Il s'adresse à quelqu'un, c'est-à-dire crée dans l'esprit de cette personne un signe 
Private piece for David Askevold (Jan. 4, 1970 "world" series): Follow-up to a re-activation of a re-activation of an activity situation using streets, travelling, following, changing 1 cation, "going wherever you go" (Street Works IV; New York City; October 3-25, 1969: "Each day, a person is chosen, at rendom, in the street, any location. I follow bim wherever he goes, no metter how long or how far he travels. I stop following only when he enters a private place (bis home, his office, etc).")

October 4: 9:25 Ali; Christopher Street and Bleecker Street, southwest corner.

A woman in a black coat; she walked east on Christopher Street, north side of the street.

At $9: 28$, she went into the A \& P, Chritopher ștreet and Seventh Avenue.

At 9:59, she left the A \& $P$, and walked west on Christopher Street.

At 10:03, she entered a building, 95 Christopher street.

November 4: The particular activity re-activated for Robert Barry ("city" series).

December 4: The particular activity and its previous re-activation re-activated for Robert Ashley ("nation" series).

Jenuary 4: The particular activity and its two previous re-activations re-activated for David Askevold.

\section{Ci-dessus}

Vito Acconci, Private Piece for David Askevold, 1969.

\section{Ci-contre}

Vito Acconci, Following Piece, 1969. 


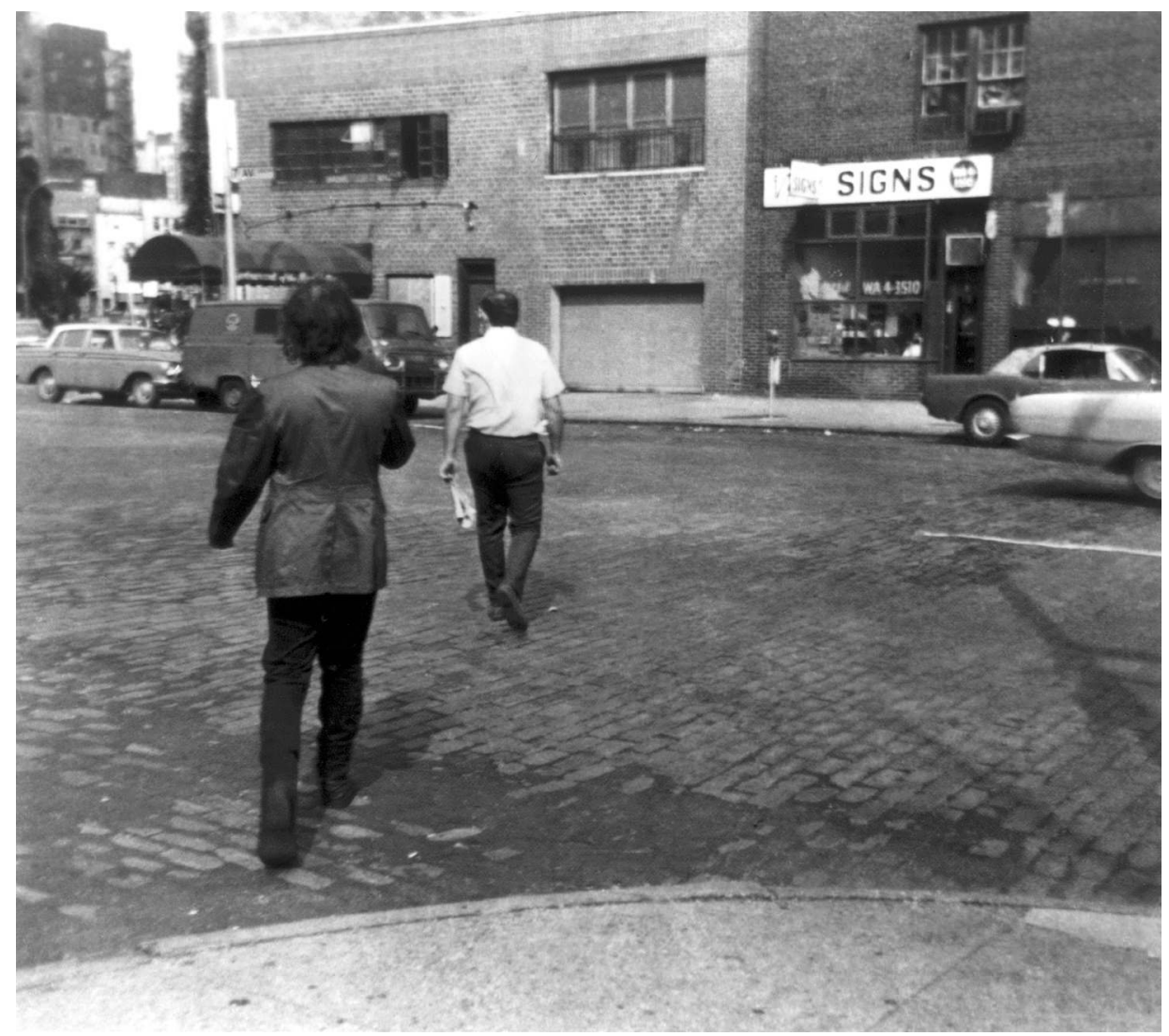




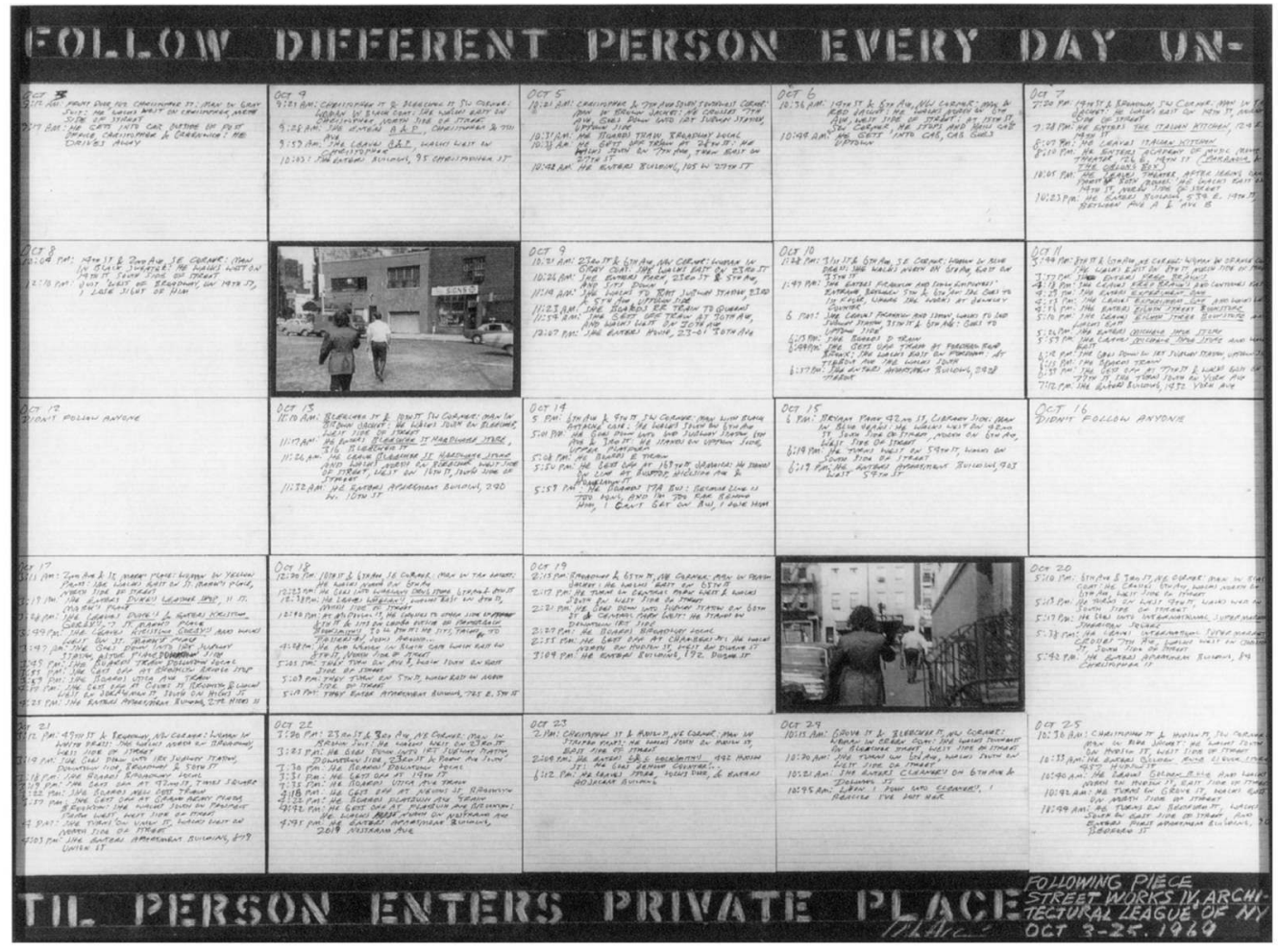

Vito Acconci, Following Piece, Documentation, 1969/1988, 1988. 
équivalent/29. ». Le signe est inséparable de son adresse et crée un autre signe (équivalent ou plus développé) dans l'esprit de la personne. Ce signe créé, Peirce l'appelle l'interprétant du premier signe, et constitue le moment de la relation signe/3o. Lorsque l'action de Vito Acconci eut lieu en octobre 1969, on suppose qu'elle fut perçue par les citadins, mais comme une activité banale de déplacement dans la ville. La signification attribuée à son geste fut celle engagée par l'habitude, soit l'interprétant logique final/31. Règle générale, l'habitude associe tel sens donné à telle chose et en cela endigue le renvoi infini d'un signe à un autre. Si le processus sémiotique est théoriquement illimité/32, dans la pratique il est cependant court-circuité par l'habitude. Les filatures se sont insérées dans l'ordre sémiotique immuable et si Acconci fut perçu, ce fut avec le regard de l'habitude, comme un simple passant.

Le sens (caché) de son acte artistique nécessite alors d'être dévoilé pour exister; il lui faut quitter l'ordre quotidien pour communiquer la nature de sa filature. Quoi de plus évident que de s'adresser directement à des personnalités du monde de l'art, en leur envoyant une lettre? Vito Acconci ne fait ensuite qu'élargir son public en produisant les photographies et le panneau (contenant tous les comptes rendus) dans un contexte artistique.

Heureux hasard ou construction délibérée, nous pouvons lire sur l'enseigne d'un magasin représenté sur la quatrième photographie de Following Piece «SIGNS ». L'artiste nous démontre-t-il à travers cette image sa façon de "faire signe »? Le dispositif créé communique à l'esprit des spectateurs les filatures et, prenant place dans un contexte apte à les recevoir, permet la venue du sens artistique. Ainsi le dispositif fait apparaître la filature comme acte potentiellement esthétique.

Or la discipline inventée par Peirce se donne pour tâche précisément de décrire l'« apparition » sémiotique, grâce à une division du signe en icône, indice et symbole. L'icône est un « signe qui renvoie à l'objet qu'il dénote simplement en vertu des caractéristiques qu'il possède, que cet objet existe réellement ou non/33 », pourvu qu'il ressemble à cette chose et soit utilisé comme signe de cette chose, sans loi ni justification. Par exemple, la photographie de l'artiste en action « ressemble » à Following Piece mais ne recueille et ne retransmet qu'un enregistrement de ce réel; il y a ici une fracture essentielle entre l'action et sa restitution (d'ailleurs complétée par le texte dans le panneau).

Au contraire, l'indice « renvoie à l'objet qu'il dénote parce qu'il est réellement affecté par cet objet/34». Ce lien physique à son objet fait l'indice. Ce rapport se pose avec une acuité plus nette dans le
/29 Charles Sanders Peirce, Écrits sur le signe, trad. G. Deledalle, Paris, Seuil, 1978, p. 140 (2.247).

/30 Au sujet de Peirce, voir Jean-Philippe Antoine, La Traversée du XXe siècle, Joseph Beuys, l'image et le souvenir, Dijon, Les Presses du réel, 2011, p. 38.

/ 31 Elle permet à deux interlocuteurs, dans un contexte précis de communication, de comprendre de quoi ils parlent, coutumiers d'attribuer telle signification à tel signe dans tel contexte.

/32 « Tout signe en tant qu'il détermine la signification d'un autre signe, devient un interprétant. Tout acte de pensée est un signe, parce qu'il est de la nature d'un interprétant [...]. ». Jean-Philippe Antoine, op. cit., p. 38.

/33 Charles Sanders Peirce, op. cit., p. 140 (2.247).

/34 ibid. 
/35 Voir à ce sujet JeanMarie Schaeffer, L'Image précaire. Du dispositif photographique, Paris, Seuil, 1987.

/36 Jean-Marie Schaeffer parle d'ailleurs d' " icône indicielle », ibid., p. 59.

/37 Charles Sanders Peirce, op. cit., p. 140 (2.249).

/38 Jean-Philippe Antoine, op. cit., p. 34 .

/39 Comme l'explique Jean-Philippe Antoine, dans le prologue de son ouvrage consacré à Joseph Beuys, l'icône s'inscrit dans le présent: la ressemblance se découvre instantanément, que l'objet existe ou non. Le symbole, lui, déploie un futur indéfini : la loi a valeur de prédiction. JeanPhilippe Antoine, op. cit.

/40 ibid., p. 44.

/41 ibid., p. 41.

/42 ibid., p. 44. cas de la photographie analogique et repose principalement sur la conscience que le spectateur a du processus mécanique de production de l'image: grâce à ses procédés chimiques et à l'action de la lumière, l'appareil photographique imprime une image du réel sur le papier photosensible/35. Empreinte de la réalité pour son récepteur, bien que non directe au sens où elle ne touche pas littéralement l'objet réel, la photographie cumule de la sorte les pouvoirs de l'indice et ceux de l'icône/36. Ajoutons qu'avec le temps, la photographie de l'artiste suivant un homme dans la rue, se dirigeant vers "Signs », est elle-même devenue le symbole de cette action pour le public averti: mille fois reproduite, elle réduit l'action à une seule image.

Enfin, concernant le panneau, l'écriture manuscrite qui couvre les 23 fiches entretient avec son auteur une relation de nature indicielle, induite par leur contiguïté. Au contraire, l'alphabet utilisé, de même que les termes employés, sont de l'ordre du symbole. Discriminatoire, le symbole nécessite une " activité préalable de l'esprit » puisqu'« il renvoie à l'objet qu'il dénote en vertu d'une loi/37 ». Le choix d'un mot exclut tous les autres possibles.

En relation avec ce qu'il représente et avec son récepteur, l'ensemble des éléments du dispositif créé par Vito Acconci cumule les trois modes de signification (icône, indice, symbole). Ces modes n'ont d'ailleurs, selon Peirce, pas d'existence séparée et n'existent que dans l'activité de relations qu'ils entretiennent. Cette triade inséparable constitue ainsi la « condition d'une pensée de l'émergence du sens dans ce qui apparaît/38 "soit, concernant Following Piece, la survenue d'une pensée critique sur l'action artistique.

Le dispositif constitué des lettres, comptes-rendus, photographies et tableaux, en révélant la filature, permet de réévaluer le passé. Il s'agit ainsi d'un effet présent, dans le présent du spectateur. Il oriente la perception de l'œuvre à rebours, rétrospectivement. Ce fonctionnement temporel particulier, cette irruption du passé dans le présent, est significatif de la dimension existentielle de l'indice. Peirce relève en effet que les trois modes de la signification se manifestent selon trois temporalités distinctes/39. Or l'indice, en connexion physique avec son objet, induit «l'idée d'un signe né directement des objets et parmi les objets/40 ». Cela suppose qu'il désigne son objet comme quelque chose «qui était déjà là avant qu'on le remarque/41", qui existait avant même la découverte de la relation par l'esprit. Ainsi l'esprit «se contenterait de prendre note après coup du fait que le réel signifie/42. ». Par analogie, nous pouvons comparer cet après coup avec la réception du dispositif par ses destinataires: le passé de l'action surgit dans le présent de la réception, comme «quelque chose » qui préexistait à son accueil. 
Nous pouvons alors qualifier ce dispositif d'indiciel, et tenter de saisir le système relationnel dans lequel il s'inscrit: réellement affecté par l'action, qui en détermine l'existence, il renvoie à son objet - la filature - comme à ce qu'il n'est pas, mais également comme à ce qui préexiste à la perception du spectateur. Trace de la connexion entre les filatures et le spectateur, le dispositif indiciel permet de faire advenir le sens artistique. Face à lui, l'expérience du spectateur n'est ni passive ni aisée; elle se conçoit dans l'adhésion à la proposition artistique. Par une sorte de pression, Following Piece rencontre ainsi son récepteur, en retard, et insiste dans son présent, pour apparaître comme œuvre.

\section{Acte, dispositif indiciel et spectateur}

Afin d'être reconnues et d'exister comme propositions symboliques, de ne pas disparaître dans l'« ici et maintenant » de leur effectuation, les filatures furtives, accomplies du 3 au 25 octobre 1969 dans les rues de New York, nécessitèrent d'être rendues publiques. Le dispositif indiciel - constitué d'éléments hétérogènes qui permettent de faire surgir le passé dans le présent - publié dans le contexte artistique, remplit alors cette fonction et fait appel à la croyance du spectateur. L'adhésion à la proposition n'est évidemment pas de même intensité selon que l'on reçoive personnellement la lettre en 1969 ou que l'on la lise au sein d'une exposition sur l'art conceptuel en 2013 (ce qui fut notre cas pour Private Piece for David Askevold/43). En effet, Vito Acconci est aujourd'hui un artiste reconnu et le musée a une plus grande puissance de légitimation que le jeune artiste.

Le contexte artistique est donc celui communément investi par le dispositif indiciel: photographies et tableaux permettent à l'artiste de s'afficher sur les cimaises des galeries tandis que les lettres, autrefois adressées à des personnalités du monde de l'art, ont aujourd'hui intégré les archives institutionnelles.

Toutefois, si l'œuvre furtive semble s'opposer au régime d'ultra visibilité, ces publicisations ne sont-elles pas paradoxales? Vito Acconci raconte aujourd'hui qu'il pensait que son travail, qui ne contenait rien de commercialisable, allait modifier le système artistique, qui lui dépendait des ventes. Or il reconnait aujourd'hui avoir eu une attitude ambivalente, qui contribua plutôt à entretenir le système qu'il dénonçait: " Je pense que nous avons rendu le système artistique encore plus puissant qu'avant: car, si effectivement notre travail ne se vendait guère, il attirait considérablement l'attention/44. ».

Ainsi Following Piece conjugue les contraires: œuvre furtive, elle attira, par son caractère insaisissable, tous les « regards », sollicités
/43 «Trafic: l'art conceptuel au Canada 1965-1980 ", exposition présentée au Badischer Kunstverein, Karlsruhe, Allemagne, avrilseptembre 2013.

/ 44 Vito Acconci, entretien avec Hans Ulrich Obrist, dans Hors Limites, l'art et la vie 1952-1994, catalogue d'exposition (Paris, Centre Georges Pompidou, 1994), Paris, Editions du Centre Pompidou, 1994, p. 307. 
par le dispositif indiciel. Ce dernier, en renvoyant à son objet, permit aux filatures de surgir dans le présent du spectateur, d'entrer dans la lumière. En se situant «contre », Vito Acconci renforçait le pouvoir de légitimation de l'institution, participant de plus à en agrandir le champ. Un tel dispositif indiciel se conformait finalement à l'attente d'un monde de l'art, lui-même indispensable à la reconnaissance symbolique de l'œuvre et sociale de l'artiste.

Cependant, avec Following Piece, Acconci construit un système complexe qui réévalue la définition de l'œuvre d'art, sa matérialité, sa temporalité, ainsi que sa réception. En effet, l'œuvre n'est plus incarnée dans un objet unique et pérenne, elle n'existe qu'au sein d'un système construit par l'acte, le dispositif indiciel et le spectateur. L'action disparaîtrait sans le dispositif, qui lui n'existerait pas sans l'activité particulière; quant à cette dualité, elle ne trouve son sens que de sa relation avec un spectateur. Le dispositif indiciel se révèle comme l'enregistrement du rapport de forces en jeu ici et indique que quelque chose de considérable a eu lieu. L'œuvre s'appréhende en retard mais s'affirme comme événement.

\section{Sophie Lapalu}

